Revista Investigaciones Turísticas, no 13, pp. 77-95

ISSN: 2174-5609

DOI. http://dx.doi.org/10.14198/INTURI2017.13.04

Tri Investigaciones

nevancostes TURISTICAS

\title{
Caracterización del turismo español según el tipo de alojamiento: aproximación mediante un modelo de gravedad
}

\author{
Luisa Martí Selva \\ Imarti@esp.upv.es \\ Rosa Puertas Medina \\ rpuertas@esp.upv.es \\ Universidad Politécnica de Valencia
}

\section{RESUMEN}

El turismo doméstico de las distintas Comunidades Autónomas (CCAA) constituye una fuente de riqueza cada vez más relevante, de ahí la importancia de realizar estudios empíricos que faciliten la adopción de políticas concretas para cada territorio. El objetivo de la investigación ha sido obtener, mediante modelos de gravedad, patrones de comportamiento de este sector con objeto de facilitar la toma de decisiones públicas y privadas que promuevan su desarrollo. El análisis se ha realizado diferenciando por tipo de alojamiento debido al perfil tan específico del turista usuario de los mismos, agrupando además las CCAA en 4 zonas según su similitud geográfica. Los resultados revelan que no es posible adoptar medidas generales para todo el territorio español, la competitividad en precios y otras variables socioeconómicas afectan de forma muy distinta a las áreas analizadas.

Palabras clave: turismo doméstico; tipo de alojamiento; modelo de gravedad 


\title{
Characterisation of Spanish tourism by type of accommodation: A gravity model approach
}

\begin{abstract}
Domestic tourism in the different Spanish Regions constitutes an increasingly relevant source of wealth, hence, the importance of empirical studies that facilitate the adoption of specific policies for each territory. Through the use of gravity models, the objective of the study is to determine the behavior patterns of this sector in order to facilitate the public and private decisions made to promote its development. The analysis has been performed by differentiating between each type of accommodation due the specific nature of the tourists who are users of these accommodations. Furthermore, the Spanish regions are grouped into four areas according to their geographical similarity. The results reveal that it is not possible to adopt general measures for the entire Spanish territory. Price competitiveness and other socioeconomic variables affect the areas analyzed very differently.
\end{abstract}

Keywords: domestic tourism; type of accommodation; gravity model

\section{INTRODUCCIÓN}

El sector turístico español ocupa una posición relevante a nivel internacional, el informe bienal emitido por el Foro Económico Mundial lo coloca, por primera vez en 2015 a la cabeza del ranking mundial en términos de competitividad (Travel \& Tourism Competitiveness Index, TTCI). Su contribución a la riqueza y desarrollo del país le ha convertido en el motor de la economía española, con un volumen de empleo superior a 2 millones de puestos de trabajo y un crecimiento del PIB en torno al 3\%. Durante los años de crisis, la industria turística ha sufrido un evidente retroceso debido a la modificación de las pautas de consumo. Sin embargo, dada su estrecha relación con la demanda externa ha podido desvincularse más fácilmente del comportamiento cíclico del resto de actividades. Además, otras situaciones de inestabilidad, conflictos, guerras en zonas con mucho atractivo turístico como la conocida "Primavera Árabe" han tenido sus efectos positivos sobre el turismo español (Perles, et al 2016).

España constituye no sólo una de las principales potencias receptoras de turistas internacionales, por detrás de Francia y EEUU, sino también de turismo interno. En 2015 tan sólo el 9,35\% de los viajes realizados por españoles tuvieron un destino fuera de las fronteras, aumentando más de un $6 \%$ los viajes nacionales realizados por residentes. El inicio de la recuperación económica está facilitando la evolución positiva de la demanda de turismo doméstico. Esta coyuntura global ha provocado un interés creciente por los estudios del comportamiento turístico.

Tal y como afirman De la Mata y Llano-Verduras (2012), a nivel español son pocos los trabajos que pueden encontrarse en la literatura donde se examina el turismo doméstico multiregional (Cañada, 2002; Millán, 2004). De ahí que la finalidad última de esta investigación ha sido tratar de aportar valores cualitativos y cuantitativos sobre un área de investigación insuficientemente explorada. En primer lugar, y diferenciando por Comunidades Autónomas (CCAA) agrupadas en cuatro áreas geográficas según su afinidad (litoral mediterráneo, costa 
verde, interior peninsular y archipiélago), se estudiará qué variables propias de estos territorios tiene mayor influencia en la elección del destino y el grado de aprovechamiento de su posición competitiva frente al resto, traduciéndose en un mayor volumen de llegadas. En segundo lugar, se tratará de analizar la existencia de patrones de comportamiento, haciendo una especial diferenciación según el tipo de acomodación elegida (hoteles, campings y alojamiento de turismo rural). El análisis se ha realizado para un periodo temporal comprendido entre 2006 y 2012, condicionado por la información estadística oficial.

El artículo se estructura en las siguientes secciones. En la sección segunda se realiza una revisión de la literatura sobre el turismo. En la sección tercera se estudia el marco de referencia del turismo de las distintas CCAA españolas. En la sección cuarta se presentan las variables y la metodología empleada. En la sección quinta se muestran los resultados obtenidos en el análisis empírico realizado. Para finalizar, en la sección sexta se aportan las principales conclusiones de la investigación.

\section{REVISIÓN DE LA LITERATURA}

El origen de la literatura relacionada con este sector económico data de los años 60 cuando, utilizando técnicas cuantitativas y cualitativas, se trataba de modelizar y predecir la demanda de esta actividad (Guthrie, 1961; Gerakis, 1965; Gray, 1966). En la última década, y dada la relación directa entre las llegadas de turistas y el crecimiento económico, las investigaciones en este área se han ido sucediendo con objeto de proporcionar elementos concretos que permitan justificar la necesidad de adoptar políticas favorables a la apertura exterior (Vogt, 2008; Arezki et al., 2009; Freytag y Vietze, 2010; entre otros). Las técnicas econométricas utilizadas se agrupan en diferentes categorías según el tipo de muestras (series temporales, panel o corte transversal e incluso muestras binarias propias de la inteligencia artificial), sin embargo no hay evidencia empírica que demuestre la superioridad de una de ellas, medida en términos de precisión (Song y Li, 2008).

Los flujos de turismo tienen un tratamiento muy similar al comercio de mercancías, donde la demanda estaría relacionada con el lugar de origen y la oferta con el destino. Esto justifica la idoneidad de utilizar modelos de gravedad para analizar los determinantes de este sector, donde las variables socioeconómicas juegan un importante papel, permitiendo igualmente diferenciar la orientación del estudio. Khadaroo y Seetanah (2008) emplean el marco gravitatorio para evaluar la importancia de las infraestructuras de transporte en la determinación del atractivo turístico de los destinos. Durbarry (2008) centra su análisis en la valoración de las implicaciones de los impuestos en la demanda de turismo inglés. Más recientemente, Fourie y Santana-Gallego (2011) tratan de cuantificar los efectos de la celebración de grandes eventos deportivos sobre las llegadas de visitantes y, Fourie y Roselló (2015) investigan el papel que juega la afiliación religiosa del país de destino en los flujos de viajeros.

Las investigaciones citadas anteriormente analizan el tránsito de turistas internacionales, sin hacer referencia a los desplazamientos nacionales. Sin embargo, los movimientos de turistas realizados dentro de un mismo territorio constituyen una fuente de riqueza nada despreciable para las distintas áreas geográficas implicadas. Existen investigaciones desarrolladas en este ámbito, siendo su objetivo primordial determinar el grado de diferenciación entre zonas para conocer el amplio universo de preferencias que condicionan los movimientos de viajeros (Eilat y Einav, 2004; Enright y Newton, 2004; Dwyer 
et al., 2009). Así, Papatheodorou (2001) llegó a la conclusión de que las características intrínsecas de los productos de ocio ofrecidos en destino constituyen la clave fundamental para la discriminación. A nivel regional, Marrocu y Paci (2013) analizan los flujos de turismo doméstico italiano, concretamente 107 provincias, haciendo especial referencia a la diferenciación del destino. Garin-Muñoz (2009) se centra en el área geográfica española al igual que De la Mata y Llano-Verduras (2012). Por su parte, Deng y Athanasopoulos (2011) investigan las áreas locales australianas y Massidda y Etzo (2012) 20 regiones italianas.

\section{MARCO DE REFERENCIA DEL TURISMO DE LAS COMUNIDADES AUTÓNOMAS ESPAÑOLAS}

El potencial económico del turismo doméstico ha avivado el interés de las CCAA por la creación de marcas propias que les identifiquen y diferencien frente al resto. El avance de las tecnologías, la aparición de nuevos destinos y alianzas entre regiones está presionando a las distintas zonas a adaptar sus productos a una coyuntura cambiante. Esta catalogación será clave para determinar y valorar la competitividad de los destinos nacionales. En España es posible distinguir entre turismo de costa, interior y rural. Tal y como estableció Reyna (1994) hace más de dos décadas, la diferencia fundamental entre los dos últimos radica en la obligatoriedad por parte del turismo rural de mantener en su desarrollo el hábitat y la actividad habitual de las áreas de destino.

La planificación turística debe girar en torno a tres cuestiones claves: el desarrollo local, la sostenibilidad ambiental y la competitividad, pudiendo medir esta última en términos de las dos primeras. La distorsión de alguna de ellas ocasionaría importantes contingencias económicas, personales y medioambientales. Ritchie y Crouch (2000) definen la competitividad como la capacidad de un país para crear valor añadido e incrementar, de esta forma, el bienestar nacional mediante la gestión de ventajas y procesos, atractivos, agresividad y proximidad, integrando las relaciones entre los mismos en un modelo económico y social.

Por otra parte, la heterogeneidad del consumo turístico ha obligado a las áreas receptoras a rediseñar sus estrategias para enfocarlas a distintos segmentos del mercado objetivo (turismo de congreso, ocio, náutico, urbano, etc.) donde su posición frente al resto pudiera ofrecer ventajas comparativas. Los movimientos turísticos están muy condicionados por factores socioeconómicos y culturales que moldean el comportamiento de los individuos y determinan la tendencia de los distintos tipos de viajes. Sin embargo, tanto la distancia como el atractivo turístico de las CCAA continúan siendo rasgos de gran influencia en los desplazamientos. Tal y como se observa en la Tabla 1, la situación económica de estos últimos años ha tenido un impacto directo sobre este sector, siendo más acusado en el turismo interno, y permaneciendo casi inalterada la proporción entre los movimientos de los residentes fuera y dentro de las fronteras. 
Tabla 1. Movimientos turísticos de los españoles según destino (valores absolutos)

\begin{tabular}{|c|c|c|c|c|c|}
\hline Año & Total & \multicolumn{2}{|c|}{ Turismo emisor } & \multicolumn{2}{c|}{ Turismo interno } \\
\hline $\mathbf{2 0 0 5}$ & 167.468 .894 & 10.463 .534 & $6,25 \%$ & 157.005 .360 & $93,75 \%$ \\
\hline $\mathbf{2 0 0 6}$ & 165.646 .233 & 10.677 .868 & $6,45 \%$ & 154.968 .365 & $93,55 \%$ \\
\hline $\mathbf{2 0 0 7}$ & 158.057 .293 & 11.275 .589 & $7,13 \%$ & 146.781 .703 & $92,87 \%$ \\
\hline $\mathbf{2 0 0 8}$ & 168.843 .820 & 11.229 .275 & $6,65 \%$ & 157.614 .545 & $93,35 \%$ \\
\hline $\mathbf{2 0 0 9}$ & 167.012 .614 & 12.017 .308 & $7,20 \%$ & 154.995 .306 & $92,80 \%$ \\
\hline $\mathbf{2 0 1 0}$ & 157.856 .547 & 12.379 .127 & $7,84 \%$ & 145.477 .420 & $92,16 \%$ \\
\hline $\mathbf{2 0 1 1}$ & 160.754 .374 & 13.346 .738 & $8,30 \%$ & 147.407 .636 & $91,70 \%$ \\
\hline $\mathbf{2 0 1 2}$ & 160.791 .418 & 12.422 .042 & $7,73 \%$ & 148.369 .376 & $92,27 \%$ \\
\hline $\mathbf{2 0 1 3}$ & 155.773 .216 & 11.245 .964 & $7,22 \%$ & 144.527 .252 & $92,78 \%$ \\
\hline $\mathbf{2 0 1 4}$ & 151.397 .320 & 11.782 .715 & $7,78 \%$ & 139.614 .605 & $92,22 \%$ \\
\hline
\end{tabular}

Fuente: Elaboración propia. Datos Familitur

Asimismo, el motivo de los desplazamientos se ha visto afectado por la coyuntura económica. A partir de 2009, el objeto "trabajo" se ve mermado por un mayor peso de las visitas a familiares o amigos, los viajes de negocios que han llegado a representar un $16 \%$ en 2007 y 2008, en los últimos años apenas superan el 5\% (Tabla 2). Ahora bien, en la medida que el crecimiento español consolide este perfil se verá nuevamente arrastrado, modificando su distribución. Por su parte, el turismo vacacional continua siendo el motor de los desplazamientos nacionales, con tasas siempre superiores al 50\% (Instituto de Turismo de España, 2012).

Tabla 2. Turismo interno según motivo

\begin{tabular}{|cccccc|}
\hline Año & Trabajo & $\begin{array}{c}\text { Visita } \\
\text { familiares/amigos }\end{array}$ & Ocio & Otros* & Total \\
\hline $\mathbf{2 0 0 6}$ & $12,2 \%$ & $28,3 \%$ & $52,1 \%$ & $7,5 \%$ & $100 \%$ \\
\hline $\mathbf{2 0 0 7}$ & $16,0 \%$ & $23,6 \%$ & $52,2 \%$ & $8,2 \%$ & $100 \%$ \\
\hline $\mathbf{2 0 0 8}$ & $16,0 \%$ & $23,6 \%$ & $52,0 \%$ & $8,4 \%$ & $100 \%$ \\
\hline $\mathbf{2 0 0 9}$ & $12,0 \%$ & $24,9 \%$ & $55,6 \%$ & $7,6 \%$ & $100 \%$ \\
\hline $\mathbf{2 0 1 0}$ & $9,8 \%$ & $27,7 \%$ & $55,1 \%$ & $7,4 \%$ & $100 \%$ \\
\hline $\mathbf{2 0 1 1}$ & $9,3 \%$ & $29,6 \%$ & $54,2 \%$ & $6,9 \%$ & $100 \%$ \\
\hline $\mathbf{2 0 1 2}$ & $7,6 \%$ & $33,3 \%$ & $52,9 \%$ & $6,2 \%$ & $100 \%$ \\
\hline $\mathbf{2 0 1 3}$ & $6,3 \%$ & $35,9 \%$ & $51,9 \%$ & $5,9 \%$ & $100 \%$ \\
\hline $\mathbf{2 0 1 4}$ & $5,3 \%$ & $34,7 \%$ & $54,3 \%$ & $5,8 \%$ & $100 \%$ \\
\hline
\end{tabular}

*Estudios, salud, religioso, entre otros

Fuente: Elaboración propia. Datos Familitur

Un análisis más detallado por CCAA perfila un comportamiento del turismo muy distinto entre ellas. Mientras que en la última década Comunidades como La Rioja, País Vasco, Murcia, Galicia, Cantabria y Baleares han experimentado una contracción de viajeros superior al $20 \%$, otras como Aragón y Navarra crecen más de un $14 \%$ y $32 \%$, respectivamente. Ello se debe al perfil cambiante y a la capacidad de adaptación de los destinos a las nuevas demandas (Gráfico 1). 
Gráfico 1. Destinos por CCAA de los movimientos turísticos de los residentes españoles (miles de personas)

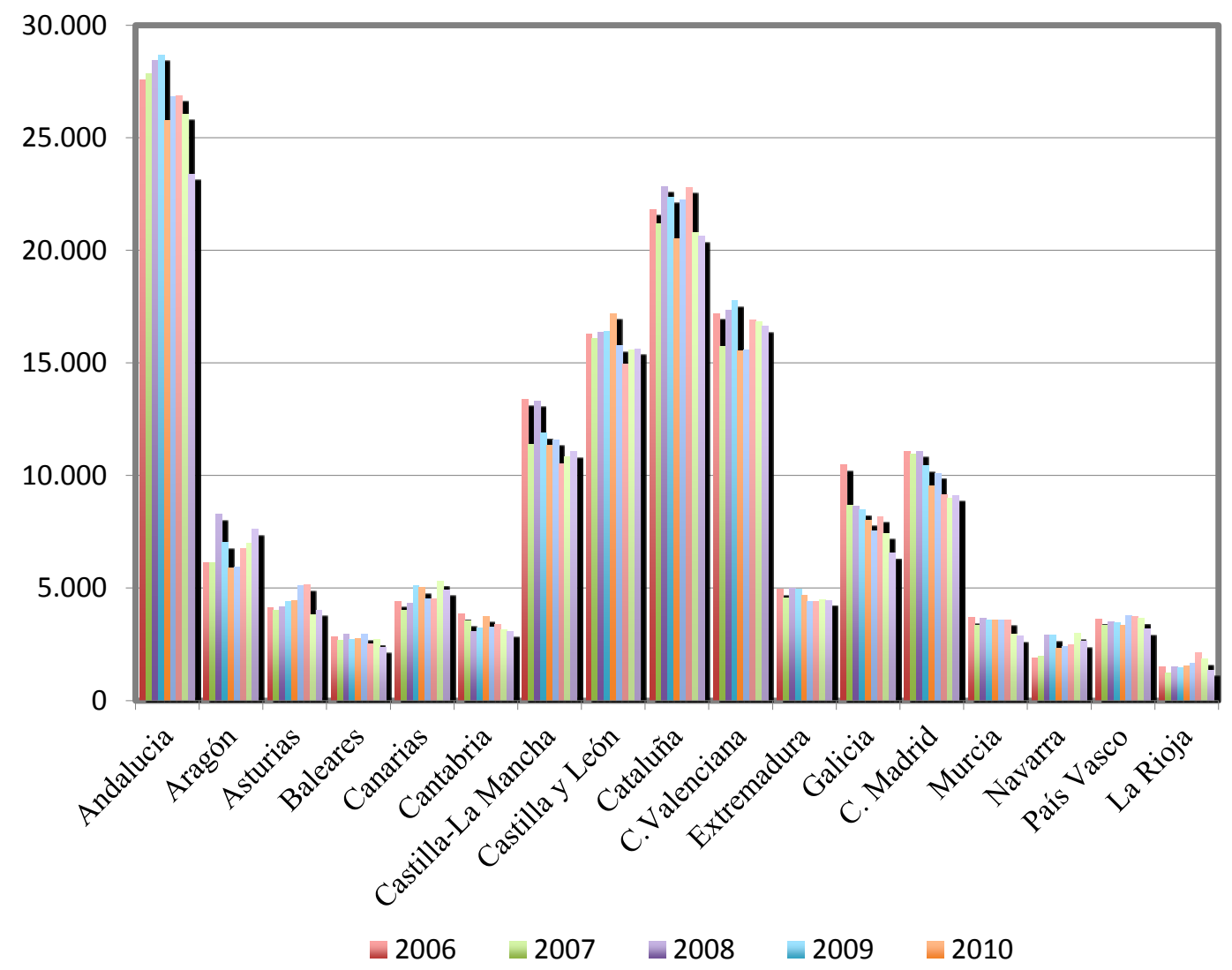

Elaboración propia. Datos Familitur.

La competitividad de precios es uno de los componentes fundamentales de la capacidad turística global de cualquier país o destino. Tal y como afirma Göral (2016), en la sociedad científica está totalmente aceptado que el precio es uno de los criterios determinantes en la toma de decisión de los viajeros. De ahí que el Instituto de Turismo de España (ITE), en un intento de aportar datos cuantitativos que permitieran determinar la posición de cada Comunidad frente al resto, ha elaborado para el periodo 2006-2012 un Índice de Tendencia de Competitividad Turística (ITCT), tomando como referencia la situación existente en 2005. Se ha construido utilizando el Índice de Precios al Consumo (IPC) del turismo y hostelería de las CCAA, ponderando con los flujos de desplazamientos entre las mismas, de manera que una disminución del índice implica una ganancia de competitividad. Los resultados reflejan que a nivel nacional, Andalucía, Galicia, Cataluña, Canarias y Comunidad Valenciana son las que ocupan siempre las primeras posiciones, frente a Navarra, La Rioja, Cantabria, Murcia y Castilla-La Mancha emplazadas en los últimos puestos.

Además, en un intento de dar homogeneidad al análisis, y siguiendo la clasificación ofrecida por el ITE (2012), las Comunidades han sido agrupadas en áreas geográficas atendiendo a la similitud de su oferta turística. Así es factible distinguir cuatro zonas:

- Litoral Mediterráneo: Cataluña, Comunidad Valenciana, Murcia y Andalucía.

- Archipiélagos: Baleares y Canarias.

- Costa Verde: Galicia, Asturias, Cantabria y País Vasco. 
- Interior Peninsular: Madrid, Castilla y León, Castilla-La Mancha, Aragón, Navarra, Extremadura y La Rioja.

Las dos primeras representan el turismo tradicional de "sol y playa", la Costa Verde se caracteriza por paisajes de montaña, cuyas Comunidades son limítrofes al litoral del mar Cantábrico, mientras que el Interior Peninsular ofrece un mayor atractivo cultural. La posición competitiva que ocupan las CCAA dentro de estas áreas no ha sufrido modificaciones significativas a lo largo del periodo analizado. En la Costa Verde es Galicia la que ocupa siempre la primera posición, siendo Aragón la correspondiente al Interior Peninsular, Cataluña y Andalucía en el Litoral Mediterráneo y Canarias en el Archipiélago.

En el Gráfico 2 se analiza la evolución de los valores del ITCT entre 2006 y 2012, para ello se ha tomado el valor medio del índice entre las CCAA de las áreas definidas previamente, es decir, para el caso del Archipiélago se ha considerado la media anual del índice para Baleares y Canarias.

Gráfico 2. Evolución de la media del ITCT según las áreas definidas

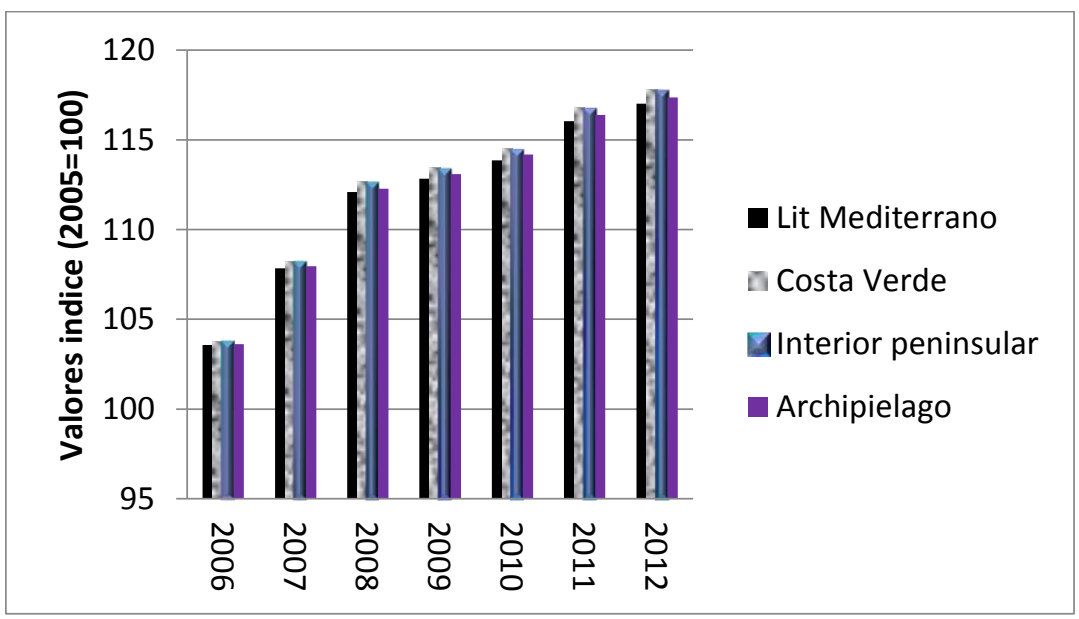

Elaboración propia a partir de los datos del ITE

La tendencia del ITCT ha sido creciente, teniendo un comportamiento similar en los grupos establecidos, caracterizado por la pérdida de competitividad a lo largo de los años. La zona de la Costa Verde resalta sobre el resto, debido en parte al mayor poder adquisitivo de sus visitantes, al tratarse de un turismo más selecto y de lujo. Por otra parte, el Archipiélago es el que ha sabido combinar adecuadamente sus incondicionales playas y paisajes con una posición de competencia en precios mejor que el resto.

Además, resulta interesante destacar el hecho de que los desplazamientos nacionales se caracterizan por realizarse dentro de la propia Comunidad y con una frecuencia elevada, cuestión apreciable en los datos disponibles para la presente investigación (Tabla 3). 
Tabla 3. Porcentaje de viajeros cuyo destino es la misma comunidad de procedencia. Por tipo de alojamiento

\begin{tabular}{|c|l|c|c|c|}
\hline Zona & \multicolumn{1}{|c|}{ Comunidad } & $\begin{array}{c}\text { Viajeros } \\
\text { Hoteles }\end{array}$ & $\begin{array}{c}\text { Viajeros } \\
\text { Campings }\end{array}$ & $\begin{array}{c}\text { Viajeros } \\
\text { Turismo } \\
\text { rural }\end{array}$ \\
\hline \multirow{3}{*}{ Litoral } & Andalucía & $53,50 \%$ & $76,64 \%$ & $6,78 \%$ \\
& Cataluña & $52,20 \%$ & $91,43 \%$ & $2,70 \%$ \\
& C. Valenciana & $31,52 \%$ & $63,14 \%$ & $1,01 \%$ \\
& Murcia & $22,9 \%$ & $50,02 \%$ & $0,71 \%$ \\
\hline \multirow{3}{*}{ Costa Verde } & Asturias & $14,39 \%$ & $29,38 \%$ & $0,12 \%$ \\
& Cantabria & $7,90 \%$ & $10,97 \%$ & $0,27 \%$ \\
& País Vasco & $22,21 \%$ & $48,86 \%$ & $33,59 \%$ \\
& Galicia & $39,50 \%$ & $67,23 \%$ & $1,83 \%$ \\
\hline \multirow{2}{*}{ Archipiélago } & Canarias & $34,72 \%$ & $99,56 \%$ & $0,30 \%$ \\
& Baleares & $22,85 \%$ & $40,83 \%$ & $0,84 \%$ \\
\hline \multirow{3}{*}{ Penterior } & Aragón & $17,57 \%$ & $32,88 \%$ & $0,44 \%$ \\
& Castilla y León & $16,90 \%$ & $22,50 \%$ & $11,31 \%$ \\
& Castilla La Mancha & $15,80 \%$ & $40,13 \%$ & $3,20 \%$ \\
& Extremadura & $3,90 \%$ & $29,90 \%$ & $0,11 \%$ \\
& Madrid & $23,26 \%$ & $81,82 \%$ & $17,75 \%$ \\
& Navarra & $8,90 \%$ & $31,58 \%$ & $0,15 \%$ \\
& La Rioja & $3,40 \%$ & $7,27 \%$ & $1,80 \%$ \\
\hline
\end{tabular}

Elaboración propia

Según el tipo de alojamiento es importante tener en cuenta a los viajeros de la misma Comunidad. Destacando sobre todo los usuarios de los campings, así por ejemplo en Canarias el 99,5\% de los clientes son oriundos del territorio, quedando en segundo lugar Cataluña cuyos residentes disfrutan igualmente de sus propios campings $(91,43 \%)$ y seguido por los madrileños $(81,82 \%)$.

Dichas proporciones se ven significativamente mermadas en todas las Comunidades si se trata de los usuarios de hoteles, al ser un alojamiento más caro y con unas características propias de un turista extranjero o desconocedor de la zona. Siguiendo con las tres Comunidades referenciadas anteriormente, en Canarias solo el $34,72 \%$ de los clientes de hoteles son de esta misma Comunidad, mientras que en Cataluña el 52,2\% y en Madrid el $23,26 \%$. La disparidad encontrada entre los usuarios de estas dos modalidades de alojamiento se podría justificar por el perfil de sus ciudadanos. Los madrileños precisan salir de la urbe propia de esta gran ciudad, están dispuestos a pasar unos días en camping aunque estén situados a pocos kilómetros de su residencia habitual simplemente por el gusto de pasar unos días en contacto con la naturaleza. Mientras que el huésped de hoteles en Madrid se ve movido por motivos de negocios o un evento concreto, reduciéndose así el número de viajeros.

Por último, los turistas de alojamiento rural casi todos eligen ubicaciones fuera de su Comunidad, comportándose de forma radicalmente distinta a los dos tipos anteriores. Destaca País Vasco con un 33,59\% y Madrid con el 17,75\%. Dichos datos evidencian que los turistas cuando se mueven dentro de su propio territorio eligen el camping o el hotel preferentemente y de forma muy residual una casa rural. 


\section{METOdologíA}

Los determinantes de los flujos de turistas domésticos se han cuantificado a través de un modelo de gravedad. Estos modelos han resurgido como una forma de modelar la demanda turística cuando se tiene que evaluar el papel de los factores estructurales en el turismo. Morley et al (2014) desarrollaron un marco teórico con especificaciones más sofisticadas que incluyen un conjunto más completo de variables explicativas y permiten la diferenciación entre países de origen y de destino. En la misma línea de ampliar los modelos de gravedad, Park y Jang (2014) demostraron que las ecuaciones de gravedad con componentes de competitividad de los destinos, como los recursos naturales y culturales, la infraestructura general y turística, la competitividad de precios y la apertura, tuvieron efectos significativos en los flujos turísticos y tenían un mayor poder explicativo que los modelos tradicionales. Sin embargo, Santeramo y Morelli (2015) han innovado en el terreno de los modelos de gravedad en la estimación aplicada a través de una regresión cuantílica para 33 países y datos entre 1998 y 2010.

En este artículo se sigue la tendencia de otros autores a tratar de ampliar los modelos tradicionales de gravedad, dado sus buenos resultados de ajuste ${ }^{1}$. De esta forma, en su especificación más básica se incluye como variables explicativas las rentas de la Comunidad de origen y de destino ( $\mathrm{PIB}$ o y $\mathrm{PIB}$ ), la población de ambos ( $\mathrm{P}_{\mathrm{O}}$ y $\mathrm{P}_{\mathrm{D}}$ ) y su distancia geográfica como proxy de los costes de transporte (D), estando su origen en las investigaciones de Tinbergen (1962) y Pöyhönen (1963a, b). Además, para analizar el nivel competitividad y otras cuestiones propias del territorio se ha utilizado tres variables adicionales: el ITCT del destino, la frontera (tener o no frontera común, $\mathrm{F}_{\mathrm{C}}$ ) y una dummy para reflejar si los movimientos se han realizado dentro de la propia Comunidad $\left(C_{P}\right)$.

La variable independiente se ha ido modificado con objeto de poder lograr los objetivos propuestos. En primer lugar, se han estimado 4 ecuaciones de gravedad para caracterizar los movimientos nacionales diferenciando por las áreas analizadas, para ello se ha utilizado como variable independiente el volumen de turistas con destino a las distintas CCAA. Posteriormente, y con objeto de detectar patrones de comportamiento dentro de cada zona geográfica se han estimado 3 ecuaciones de gravedad discriminando según el hospedaje elegido por el turista (hotel, camping o turismo rural). Todo ello ha exigido, modificar las variables dependientes e independientes adaptándolas a cada estudio particular. La expresión general es la siguiente:

$$
\begin{aligned}
& \log \left(T_{i j}\right)=\beta_{0}+\beta_{1} \log \left(D_{i j}\right)+\beta_{2} \log \left(P_{O} B_{O}\right)+\beta_{3} \log \left(P_{D} B_{D}\right)+\beta_{4} \log \left(P_{O}\right)+\beta_{5} \log \left(P_{D}\right)+\beta_{6} \\
& I T C T_{D}+\beta_{7} C C A A+\beta_{8} \text { Frontera }+u_{i j}
\end{aligned}
$$

Donde,

$\mathrm{T}_{\mathrm{ij}}$ : Flujos de turistas de la Comunidad Autónoma i a la j

$D_{\mathrm{ij}}$ : Distancia entre la Comunidad Autónoma i y la j

PIBo: PIB de la Comunidad de origen

$\mathrm{PIB}_{\mathrm{D}}$ : PIB de la Comunidad de destino

Po: Población de la Comunidad de origen

$\mathrm{P}_{\mathrm{D}}$ : Población de la Comunidad de destino

ITCT $\mathrm{D}$ : Índice de tendencia de competitividad turística de la Comunidad de destino

\footnotetext{
${ }^{1}$ Lorde y Airey (2015) también ampliaron un modelo de gravedad para analizar el turismo en el Caribe.
} 
CCAA: variable ficticia que toma el valor 1 si es un turista que viaja dentro de su Comunidad y 0 en caso contrario

Frontera: variable ficticia que toma el valor 1 si la Comunidad i tiene una frontera común con j.

En este planteamiento se parte de la hipótesis de que las variables incorporadas tienen un impacto significativo en los flujos de turistas nacionales y los signos son coherentes según los postulados de la teoría económica. La distancia, como indicativa de los costes de transporte, no está exenta de problemas al suponer que los mismos son independientes del medio utilizado y las capitales de cada Comunidad los centros turísticos de la misma. Su efecto entre Comunidades $\left(\beta_{1}\right)$ debe ser negativo, porque la cercanía promueve un mayor número de turistas.

Teóricamente, los coeficientes del PIB, tanto del origen como del destino $\left(\beta_{2}\right.$ y $\left.\beta_{3}\right)$ serán positivos, a mayor volumen económico es de esperar que la gente tenga más predisposición a viajar y la Comunidad a recibir turistas. Por otra parte, el coeficiente de la población para la Comunidad de origen $\left(\beta_{4}\right)$ debe de ser positivo, indicando que existe un universo más amplio de individuos potenciales a desplazarse a otros territorios. Sin embargo, el coeficiente de la población de destino $\left(\beta_{5}\right)$ puede tener un signo ambiguo, en caso de ser positivo el turista será atraído por destinos masificados donde encuentra su diversión. En cambio, si es negativo se está ante un turista que huye de los lugares bulliciosos, buscando parajes tranquilos y poco poblados.

De acuerdo con el objetivo del artículo, se incluye en el modelo de gravedad el valor del ITCT para la Comunidad de destino, el cual lleva asociado un coeficiente $\left(\beta_{6}\right)$ que debe ser negativo, ya que un mayor valor del índice indica que el territorio es menos competitivo en precios y, por tanto, atraerá un número reducido de turistas. Por último, el conjunto de variables ficticias representan las similitudes sociales y culturales existentes entre los países de las áreas geográficas analizadas y deberán llevar un coeficiente con un signo positivo, ya que la gente tiende a viajar por su propia Comunidad o por una muy próxima donde hay una frontera que las separa.

La información relativa a los flujos de turistas, el PIB y la población proceden de la base de datos publicada por el Instituto Nacional de Estadística español (INE). El índice de competitividad se ha tomado de la publicación realizada por el ITE en 2012. En cuanto a la distancia entre CCAA expresada en kilómetros, se ha obtenido de la web "http://www.distanciasentreciudades.com/espana"2 sirviendo como una primera aproximación dada la complejidad de determinar el punto exacto de salida y llegada. Finalmente, el conjunto de variables ficticias ha sido elaborado por los propios autores.

\section{RESULTADOS}

El estudio empírico se ha realizado a partir de la estimación de la ecuación (1) con datos panel para el periodo 2006-2012, agrupando las CCAA en cuatro zonas según su situación geográfica. Posteriormente, y diferenciando por tipo de alojamiento, se ha especificado esta ecuación adaptándola a los objetivos de la investigación. Todos los coeficientes de las estimaciones realizadas han sido estandarizados, con el propósito de eliminar las unidades de

${ }^{2}$ Los datos de distancias son calculados a partir de API de Google Maps 
medida de las variables y poder realizar un análisis comparativo de todas las sensibilidades obtenidas.

Los resultados de la estimación de datos panel mediante efectos aleatorios ${ }^{3}$, incluyendo los tres tipos de alojamiento según la zona geográfica donde se ubiquen, se recogen en la Tabla 4, donde se comienza a caracterizar cada área por sus propias particularidades ${ }^{4}$.

Tabla 4. Resultados de la estimación para el conjunto de viajeros

\begin{tabular}{|c|c|c|c|c|}
\hline & $\begin{array}{c}\text { Litoral } \\
\text { Mediterráneo }\end{array}$ & Costa Verde & Archipiélago & $\begin{array}{c}\text { Interior } \\
\text { Peninsular }\end{array}$ \\
\hline $\mathrm{Po}_{\mathrm{O}}$ & $0,424 * * *$ & $0,378 * * *$ & $0,471 * * *$ & $0,365^{* * *}$ \\
\hline$P_{D}$ & $0,410 * * *$ & $-0,027$ & $-0,258 * * *$ & $0,270 * * *$ \\
\hline $\mathrm{D}$ & $-0,226 * * *$ & $-0,246 * * *$ & $-0,050$ & $-0,173 * * *$ \\
\hline $\mathrm{PIB}_{\mathrm{O}}$ & $0,075 * * *$ & $0,176 * * *$ & $0,145 * * *$ & $0,141^{* * *}$ \\
\hline $\mathrm{PIB}_{\mathrm{D}}$ & $-0,056^{* *}$ & $-0,118 * * *$ & $-0,176 * * *$ & $-0,036$ \\
\hline ITCT & $-0,010$ & $-0,035 * * *$ & $-0,013$ & $-0,008$ \\
\hline$C_{P}$ & $0,122 * * *$ & $0,073 * * *$ & $0,295^{* * *}$ & $0,148^{* * *}$ \\
\hline $\mathrm{F}_{\mathrm{C}}$ & $0,079 * *$ & $0,060 * *$ & ND & $0,163 * * *$ \\
\hline № obs & 476 & 357 & 238 & 952 \\
\hline № grupos & 68 & 51 & 34 & 136 \\
\hline
\end{tabular}

En todas las estimaciones la variable más influyente en la determinación de la cantidad de viajeros es la población de origen, incluso por delante de la riqueza, indicando que a mayor volumen de habitantes más desplazamientos. En una posición similar se encuentra la población de destino, cuyo comportamiento está condicionado por los territorios analizados. Según la teoría económica, el signo de este coeficiente es ambiguo, y de esta forma se identifican los primeros rasgos distintivos de las CCAA analizadas. Si es positivo, se trata de un turismo ligado a la masificación como ocurre Litoral Mediterráneo y el Interior Peninsular $(0,410$ y 0,270$)$. Por el contrario, el signo negativo representa que el turista nacional busca la tranquilidad en sus vacaciones, como es el caso de Baleares y Canarias $(-0,258)$, donde la elevada aglomeración está restando afluencia española a las Islas.

La tercera variable en importancia es la distancia, su coeficiente negativo implica que la lejanía del destino lleva asociado un encarecimiento del viaje y, por tanto, resta afluencia a dichos lugares. Nuevamente, las Islas tienen un comportamiento singular, pues la proximidad no resulta determinante en el volumen de sus visitantes. Este resultado en ningún caso contradice la lógica económica y social, la mayor o menor distancia de los desplazamientos desde la península no supone una barrera a la llegada de visitantes a Baleares y Canarias.

Por otra parte, el factor riqueza de la Comunidad de origen es influyente en el número de viajeros siendo su coeficiente positivo y significativo para todas las zonas analizadas.

\footnotetext{
${ }^{3}$ En la tabla A1 del anexo se exponen los resultados de la estimación por pooled MCO, efectos aleatorios y efectos fijos. Y tanto por el test F, el test de Breusch y Pagan como por el test de Hausman es preferible la estimación por efectos aleatorios. Los coeficientes de la tabla del anexo no coinciden con los de la tabla 4 por estar estandarizados en esta última.

${ }^{4}$ Los errores standard de las estimaciones son clásicos MCO.
} 
Siguiendo los postulados básicos de la teoría microeconómica, y dada la elevada elasticidad renta de un bien de ocio como es el turismo, es coherente el signo obtenido, el mayor poder adquisitivo se traduce en más movimientos de viajeros. Mientras que en destino la riqueza es susceptible de traducirse en mayor calidad del servicio a precios elevados, pudiendo restar atractivo a la zona.

El hecho de pertenecer a la misma CCAA tiene más importancia en el Archipiélago que en el resto de zonas por ser un destino alejado de la península. Los residentes insulares tienen menos opciones al tener que recorrer largas distancias para salir de su Comunidad. El otro determinante cualitativo, tener frontera común entre el origen y el destino del viajero, resulta más determinante en el Interior Peninsular debido fundamentalmente al número elevado de CCAA colindantes.

Por último, el ITCT tan solo resulta significativo en Costa Verde, indicando que para el conjunto de viajeros con destino el País Vasco, Cantabria y Asturias sí es importante la competitividad en precios, es decir, un aumento de dicho índice disminuiría la cantidad de viajeros. En las demás zonas no es influyente, siendo el resto de variables las decisivas para determinar el volumen nacional de viajeros.

Siguiendo con el objetivo del trabajo, se ha buscado caracterizar los destinos según el tipo de estancia elegido. Con este fin, se han realizado estimaciones del modelo de gravedad diferenciando por tipo de alojamiento (hoteles, campings y turismo rural) en cada una de las cuatro zonas analizadas (Litoral Mediterráneo, Costa Verde, Archipiélago e Interior Peninsular). En primer lugar, se presentan en la Tabla 5 los resultados obtenidos para el Litoral Mediterráneo.

Tabla 5. Litoral Mediterráneo

\begin{tabular}{|l|c|c|c|}
\hline & Hoteles & Camping & $\begin{array}{c}\text { Turismo } \\
\text { rural }\end{array}$ \\
\hline $\mathrm{P}_{\mathrm{O}}$ & $0,384^{* * *}$ & $0,381^{* * *}$ & $0,453^{* * *}$ \\
$\mathrm{P}_{\mathrm{D}}$ & $0,453^{* * *}$ & $0,382^{* * *}$ & $0,308^{* * *}$ \\
$\mathrm{D}$ & $-0,175^{* * *}$ & $-0,196^{* * *}$ & $-0,208^{* * *}$ \\
$\mathrm{PIB}_{\mathrm{O}}$ & $0,036^{* * *}$ & $0,151^{* * *}$ & $0,138^{* * *}$ \\
$\mathrm{PIB}_{\mathrm{D}}$ & $0,039^{* * *}$ & $-0,003$ & $-0,029$ \\
$\mathrm{ITCT}$ & $-0,035^{* * *}$ & $-0,045^{* * *}$ & $-0,001$ \\
$\mathrm{C}_{\mathrm{P}}$ & $0,088^{* * *}$ & $0,189^{* * *}$ & $0,190^{* * *}$ \\
$\mathrm{~F}_{\mathrm{C}}$ & $0,047^{* * *}$ & $0,228^{* * *}$ & $0,171^{* * *}$ \\
\hline
\end{tabular}

Nota: ${ }^{* * *} \mathrm{p}$-value $<0,01$

Elaboración propia

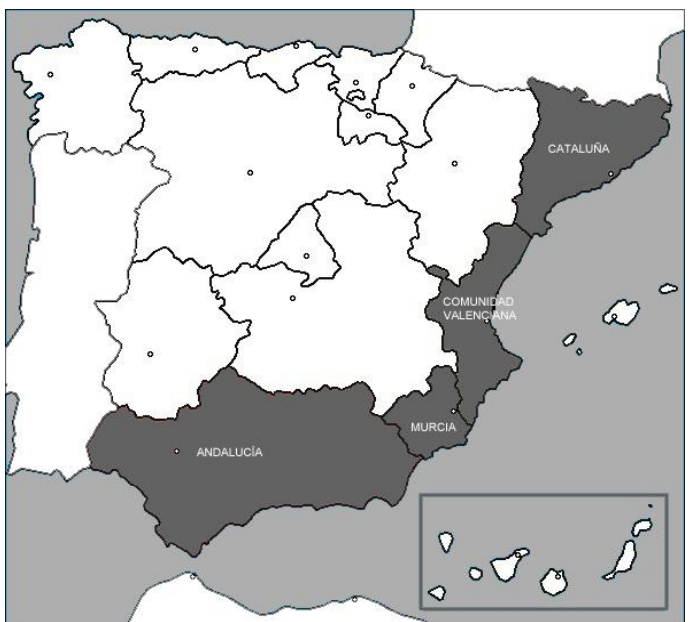

Tal y como se puede observar, los determinantes del volumen de turistas en esta área geográfica no presentan grandes diferencias según modalidad de estancia elegida. En todos los casos, se trata de turistas atraídos por la densidad de población en destino, siendo la mayor población de origen un condicionante también positivo para una mayor afluencia. La distancia es significativa y negativa, ligado a este resultado se encuentra el hecho de que volumen de turistas cuyo destino es el Litoral Mediterráneo aumenta si éstos pertenecen a alguna Comunidad de este territorio y si además existe frontera común entre origen y destino. 
Las variables que permiten discriminar entre el tipo de alojamiento elegido es la riqueza de origen y destino, y la competitividad. EI PIB de destino solo es significativo para los usuarios de hoteles, circunstancia que podría explicarse por el hecho de que la mayor renta de estas Comunidades se traduce en mejores servicios e infraestructuras hoteleras, atrayendo a un número más elevado de visitantes. Por el contrario, el PIB de origen es significativo y positivo en los tres casos, siendo más relevante en el turismo rural, cuestión que a priori no se hubiera aventurado. Respecto a la competitividad también surgen diferencias, el ITCT es significativo y negativo en el caso de que los viajeros elijan hospedarse en hoteles y campings, es decir, la mayor competitividad se traducirá en más huéspedes, siendo su peso muy inferior al resto de variables analizadas.

Conclusiones muy distintas se obtienen cuando se analizan las estimaciones realizadas para los viajeros con destino Costa Verde (Tabla 6).

Tabla 6: Costa Verde

\begin{tabular}{|l|c|c|c|}
\hline & Hoteles & Camping & $\begin{array}{l}\text { Turismo } \\
\text { rural }\end{array}$ \\
\hline Po $_{\mathrm{O}}$ & $0,362^{* * *}$ & $0,385^{* * *}$ & $0,380^{* * *}$ \\
$\mathrm{P}_{\mathrm{D}}$ & $0,087^{* * *}$ & $-0,054$ & $-0,046$ \\
$\mathrm{D}$ & $-0,257^{* * *}$ & $-0,414^{* * *}$ & $-0,248^{* * *}$ \\
$\mathrm{PIB}_{\mathrm{O}}$ & $0,054^{* * *}$ & $0,127^{* * *}$ & $0,188^{* * *}$ \\
$\mathrm{PIB}_{\mathrm{D}}$ & $-0,019$ & $-0,195^{* * *}$ & $-0,136^{* *}$ \\
$\mathrm{ITCT}^{* *}$ & $-0,013^{* * *}$ & $-0,037^{* * *}$ & $-0,049^{* * *}$ \\
$\mathrm{C}_{\mathrm{P}}$ & $0,049^{* *}$ & $0,082^{* *}$ & $0,067^{* * *}$ \\
$\mathrm{~F}_{\mathrm{C}}$ & $0,049^{*}$ & 0,071 & $0,051^{*}$ \\
\hline
\end{tabular}

Nota: ${ }^{*} \mathrm{p}$-value $<0,1,{ }^{* *} \mathrm{p}$-value $<0,05,{ }^{* * *} \mathrm{p}$-value $<0,01$; Elaboración propia

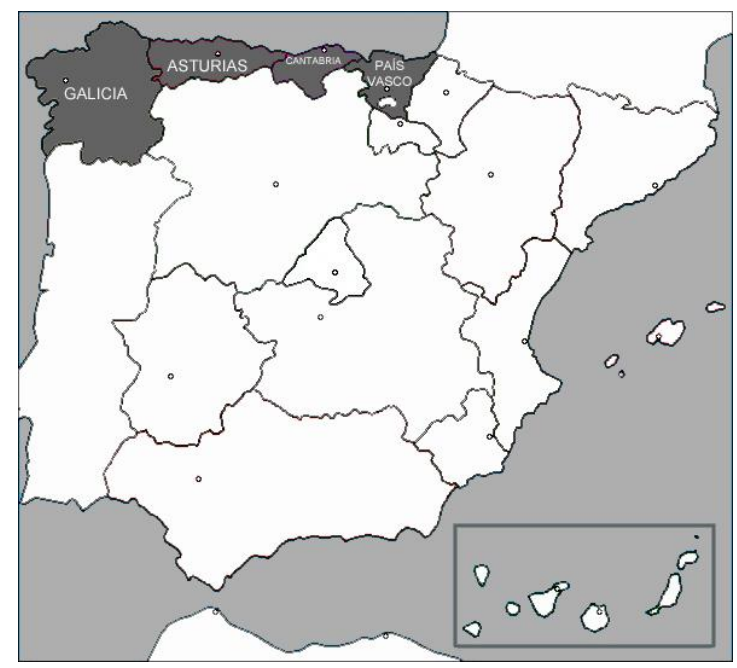

Las variables de mayor peso son la población de origen y la distancia, destacando que los turistas alojados en camping se ven más afectados por la distancia que por su nivel de renta. Además, se observa que la población de destino tan sólo afecta a los que eligen hoteles, siendo indiferente en el caso del camping y turismo rural. Otra singularidad de esta área geográfica es el hecho de que los viajeros alojados en camping y áreas rurales buscan zonas de menor renta $(-0,195$ y $-0,136$, respectivamente) debido fundamentalmente al menor precio de sus servicios, hecho que viene indicado por el signo negativo del coeficiente del PIB de destino. Además, el nivel de competitividad afecta levemente a los viajeros independientemente del tipo hospedaje, al igual que variables cualitativas, salvo en el caso de los alojados en camping que optan en mayor medida por visitar Comunidades colindantes a la suya.

A continuación, se muestran los resultados obtenidos para los turistas de Canarias y Baleares (Tabla 7). 
Tabla 7. Archipiélagos

\begin{tabular}{|c|c|c|c|}
\hline & Hoteles & Camping & $\begin{array}{c}\text { Turismo } \\
\text { rural }\end{array}$ \\
\hline$P_{0}$ & $0,466^{* * *}$ & $0,300^{* * *}$ & $0,480^{* * *}$ \\
\hline$P_{D}$ & $-0,205^{* * *}$ & $-0,397^{* * *}$ & $-0,313^{*}$ \\
\hline $\mathrm{D}$ & $-0,041$ & $-0,369^{* * *}$ & $-0,099$ \\
\hline $\mathrm{PIB}_{0}$ & $0,140^{* * *}$ & $0,187^{* * *}$ & $0,238^{* * *}$ \\
\hline $\mathrm{PIB}_{\mathrm{D}}$ & $-0,109^{* * *}$ & $-0,100$ & $-0,286^{* * *}$ \\
\hline ITCT & $-0,020^{* *}$ & 0,017 & 0,009 \\
\hline$C_{P}$ & $0,271^{* * *}$ & $0,377^{* * *}$ & $0,338^{* * *}$ \\
\hline$F_{c}$ & ND & ND & ND \\
\hline
\end{tabular}

Elaboración propia

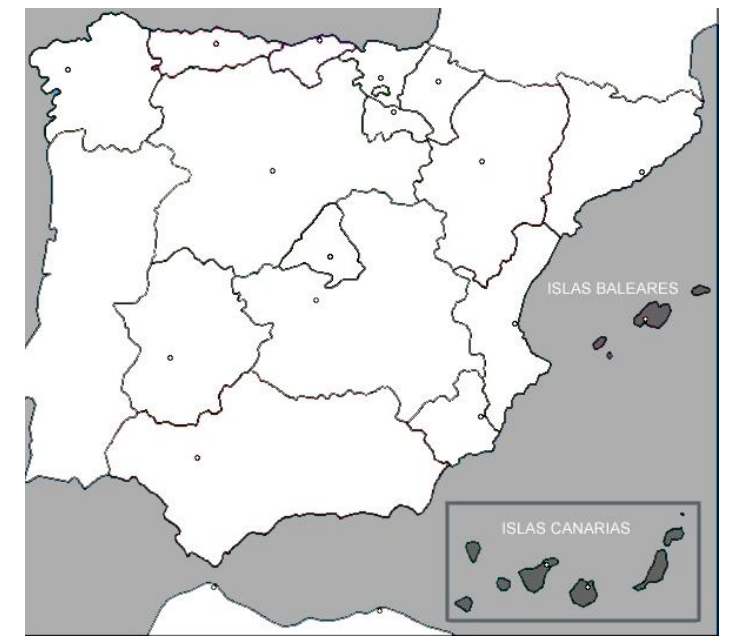

Se trata de Comunidades muy pobladas en temporadas altas de turismo, de ahí que esta masificación, independientemente del alojamiento elegido, esté restando afluencia nacional, aun tratándose de un destino de "sol y playa". Mientras que la distancia tan solo afecta negativamente a aquellos visitantes que optan por pernoctar en camping, cuyo poder adquisitivo es menor. El resto, elegido este destino, no está condicionado por el coste del viaje traducido en cantidad de kilómetros a realizar, en muchos casos elevados al pasar de la península a las Islas.

Al igual que en las otras áreas analizadas el PIB de origen y destino son determinantes del volumen de turistas, de forma directa e indirecta, respectivamente, salvo en el caso del camping cuyo PIB de destino no resulta significativo. Por otra parte, la cualidad de pertenecer a la misma CCAA es muy significativa, para un nativo de las Islas es mucho más fácil y barato moverse dentro de su propia Comunidad, y no viajar hasta la península, cuyo trayecto inevitablemente encarecería el desplazamiento. Otra cuestión importante es que el ITCT solo condiciona a los que deciden hospedarse en hoteles, donde una pérdida de competitividad ocasionada por un incremento del precio afectaría negativamente al volumen de visitantes a las Islas.

Finalmente se analizan los determinantes de los viajeros con destino el Interior Peninsular (Tabla 8). 
Tabla 8. Interior Peninsular

\begin{tabular}{|l|c|c|c|}
\hline & Hoteles & Camping & $\begin{array}{l}\text { Turismo } \\
\text { rural }\end{array}$ \\
\hline $\mathrm{P}_{\mathrm{O}}$ & $0,339^{* * *}$ & $0,373^{* * *}$ & $0,387^{* * *}$ \\
$\mathrm{P}_{\mathrm{D}}$ & $0,371^{* * *}$ & $0,132^{* * *}$ & $0,185^{* * *}$ \\
$\mathrm{D}$ & $-0,171^{* * *}$ & $-0,213^{* * *}$ & $-0,171^{* * *}$ \\
$\mathrm{PIB}_{\mathrm{O}}$ & $0,043^{* * *}$ & $0,081^{* * *}$ & $0,165^{* * *}$ \\
$\mathrm{PIB}_{\mathrm{D}}$ & $0,048^{* * *}$ & $-0,040$ & $-0,120^{* * *}$ \\
$\mathrm{ITCT}$ & $-0,020^{* * *}$ & $-0,001$ & $-0,005$ \\
$\mathrm{C}_{\mathrm{P}}$ & $0,083^{* * *}$ & $0,211^{* * *}$ & $0,154^{* * *}$ \\
$\mathrm{~F}_{\mathrm{C}}$ & $0,065^{* * *}$ & $0,210^{* * *}$ & $0,203^{* * *}$ \\
\hline \multicolumn{4}{|c|}{ Nota: ${ }^{* *} \mathrm{p}$-value<0,05, ${ }^{* * *} \mathrm{p}$-value<0,01 } \\
\hline
\end{tabular}

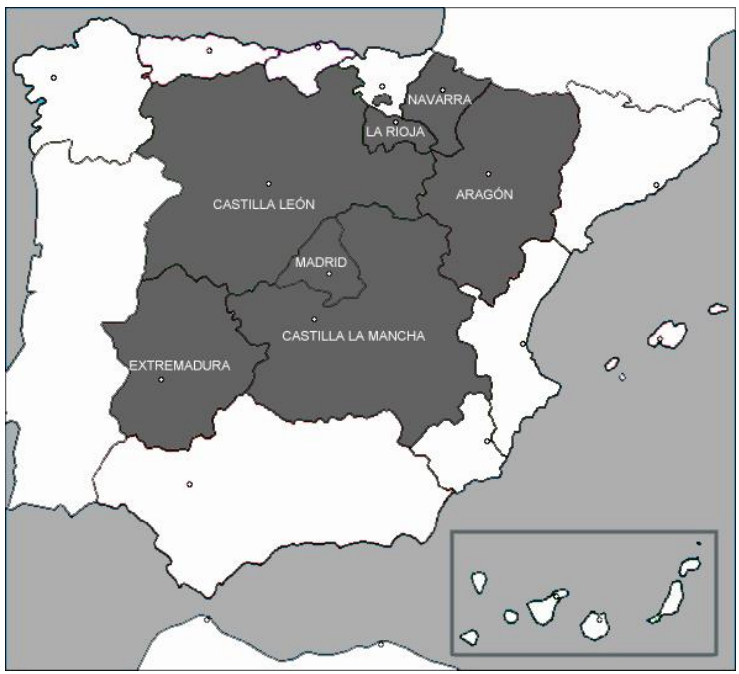

De forma similar a las anteriores estimaciones, las variables población de origen y destino son las más influyentes. Al tratarse de CCAA de menor afluencia turística y, en términos generales, menos pobladas, el volumen de habitantes afecta positivamente a la cantidad de personas que deciden pasar unos días en estas Comunidades.

La distancia se comporta de forma coherente con la teoría del modelo siendo más influyente para los usuarios de campings. También, hay que destacar el mayor peso del coeficiente de la dummy frontera frente a las otras zonas analizadas, al tratarse de una zona con muchas Comunidades limítrofes los movimientos de personas se ven atraídos por sus vecinos más próximos. En cuanto a la competitividad, hay que destacar que nuevamente al igual que ocurre en las Islas, tan sólo es significativo para aquellos turistas que deciden alojarse en hoteles. Una bajada de las tarifas hoteleras conllevaría una mayor afluencia a esta área territorial.

\section{CONCLUSIONES}

La información estadística demuestra que el turismo doméstico es mucho más relevante que el internacional, sus movimientos son más difíciles de analizar y caracterizar, existiendo escasez de trabajos que profundicen en este ámbito, de ahí la importancia del estudio realizado. Se trata de una parte del sector terciario, muy sensible a la estacionalidad deseosa de buscar nuevos nichos de mercado que permita aumentar la afluencia fuera de los meses vacacionales.

La investigación realizada se ha centrado en turistas que han sido usuarios de hoteles, campings o alojamientos rurales, todos ellos bajo una denominación de establecimientos reglados que suponen la masa gruesa de viajeros entre las regiones españolas. No obstante, la existencia de establecimientos no reglados es conocida por todos haciendo reflexionar en el hecho de que determinadas conclusiones no se podrían generalizar al tipo de turistas que suelen utilizar dichos establecimientos.

El estudio realizado ha caracterizado a los destinos turísticos desde el punto de vista del consumidor nacional, siendo la población y el PIB de destino las variables discriminadoras 
del alojamiento elegido en cada zona. En las CCAA costeras pertenecientes al Litoral Mediterráneo y al Archipiélago, ambas caracterizadas por un turismo mayoritariamente de "sol y playa", a priori podrían esperarse comportamientos muy similares. Sin embargo, los resultados revelan que, con independencia del hospedaje elegido, en las Islas la aglomeración de gente resta volumen turístico, ocurriendo lo contrario en la zona mediterránea. Igualmente, el signo negativo del PIB de destino obtenido en hoteles y turismo rural de las Islas contradice, el signo positivo de los alojados en hoteles del Litoral. Cada área geográfica tiene un patrón de comportamiento distinto que debería analizarse en profundidad para evitar medidas económicas que se alejen de sus necesidades.

Los gobiernos de las distintas Comunidades tienen que favorecer la oferta de paquetes turísticos atractivos que suavicen el efecto negativo causado por la lejanía a otros territorios. Políticas que favorezcan la modernización de infraestructuras y mejoren el transporte, abaratando los desplazamientos, permitirían minimizar esta situación impulsando la mayor entrada de visitantes. Del mismo modo, las Islas necesitan establecer normas para controlar el volumen de llegadas de tal forma que en ningún caso la masificación reste afluencia. Se deberían focalizar los esfuerzos en intentar atraer a turistas de calidad que incrementen la entrada de riqueza en la Comunidad, sustituyendo a aquellos cuyo gasto en destino es muy reducido.

El turismo es una fuente importante de ingresos, empleo, así como recaudación de impuestos para las zonas de destino. Sin embargo, las autoridades locales en ocasiones no valoran el hecho de que la imposición fiscal en determinados servicios eleva en gran medida los precios, con la consiguiente pérdida de competitividad y reducción, por tanto, del número de visitantes. Con independencia del destino elegido, los alojados en hoteles tienen una gran dependencia de esta variable (ITCT), de forma que las políticas fiscales expansivas que permitieran reducir el precio de este tipo de hospedaje se traducirían en un mayor movimiento de personas suponiendo una importante fuente de ingresos y empleo para el territorio.

Las áreas turísticas necesitan realizar un análisis comparativo basado en la competitividad de precios de sus principales rivales, dada la importancia de este factor en el proceso de toma de decisiones de los viajeros. Sin embargo, y como ampliación a los resultados aportados, las CCAA deberían disponer de una valoración de la competitividad global del destino, dado que existen otros factores que también afectan a la elección del viaje.

\section{REFERENCIAS BIBLIOGRÁFICAS}

Arezki, R., Cherif, R. y Piotrowski, J. (2009). Tourism Specialization and Economic Development: Evidence from the UNESCO World Heritage List, IMF Working Paper, WP/09/176.

Cañada, A. (2002). El turismo interior en España y su dimensión espacial: las regiones como exportadoras e importadoras de turismo interior. Cuadernos de Información Económica, 169, 137-140.

De la Mata, T. y Llano-Verduras, C. (2012). Spatial pattern and domestic tourism: an econometric analysis using inter-regional monetary flows by type of journey. Papers in Regional Science, 91(2), 437-470.

Deng, M. y Athanasopoulos, G. (2011). Modelling Australian domestic and international inbound: a spatial-temporal approach. Tourism Management, 32, 1075-1084. 
Durbarry, R. (2008). Tourism Taxes: Implications for Tourism Demand in the UK. Review of Development Economics, 12 (1), 21-36.

Dwyer, L., Edwards, D., Mistilis, N.; Roman, C. y Scott, N. (2009). Destination and enterprise management for a tourism future. Tourism Management, 30, 63-74.

Eilat, Y. y Einav, L. (2004). Determinant of international tourism: a three dimensional panel data analysis. Applied Economics, 36, 1315-1327.

Enright, M.J. y Newton, J. (2004). Tourism destination competitiveness: a quantitative approach. Tourism Management, 25, 777-788.

Freytag, A. y Vietze C. (2010). Can Nature Promote Development? The Role of Sustainable Tourism for Economic Growth. Jena Economic Research Paper 08/2010, 1-32.

Fourie, J. y Rosselló, J. (2015). Religion, Religious Diversity and Tourism. Kyklos, 68(1), 51-64.

Fourie, J. y Santana-Gallego, M. (2011). The impact of mega-sport events on tourist arrivals. Tourism Management, 32, 1364-1370.

Garin-Muñoz, T. (2009). Tourism in Galicia: domestic and foreign demand. Tourism Economics, 15, 753-769.

Gerakis, A.S. (1965). Effects of Exchange-Rate Devaluations and Revaluations on Receipts from Tourism. International Monetary Fund Staff Papers, 12, 365-384.

Göral, R. (2016). Inernational Tourism Price Competitive Index and a Comparative Application with Turkey. International Journal of Scientific and Research Publications, 7(4), 308-313.

Gray, H.P. (1966). The Demand for International Travel by United States and Canada. International Economic Review, 7, 83-92.

Guthrie, H.W. (1961). Demand for Tourists' Goods and Services in a World Market. Papers and Proceedings of the Regional Science Association, 7, 159-175.

Instituto de Turismo de España. (2012). Movimientos turísticos de los españoles (FAMILITUR). Informe anual. Ministerio de Industria, Energía y Turismo.

Khadaroo, J. y Seetanah, B. (2008). The role of transport infrastructure in international tourism development: A gravity model approach. Tourism Management, 29, 831-840.

Lorde, Li y Airey (2015). Modeling Caribbean Tourism Demand: An Augmented Gravity Approach. Journal of Travel Research, 55 (7), 946-956.

Marrocu, E. y Paci, R. (2013). Different tourists to different destinations. Evidence form spatial interaction models. Tourism Management, 39, 71-83.

Massidda, C. y Etzo, L. (2012). The determinants of Italian domestic tourism: a panel data analysis. Tourism Management, 33, 603-610.

Millán, M. (2004). Turismo en la Región de Murcia: evolución de la oferta turística de interior y su distribución espacial. Cuadernos de Turismo, 13, 51-72.

Morley, C., Rosselló, J. y Santana-Gallego, M. (2014). Gravity models for tourism demand: theory and use. Annals of Tourism Research, 48, 1-10.

Papatheodorou, A. (2001). Why people travel to different places. Annals of Tourism Research, 28, 164-179.

Park, J.-Y. y Jang, S. (2014). An Extended Gravity Model: Applying Destination Competitiveness. Journal of Travel \& Tourism Marketing, 31, 799-816.

Perles, J.F., Ramón, A.B., Moreno, L. y Torregrosa, M.T. (2016). Winners and losers in the Arab uprisings: a Mediterranean tourism perspective. Current issues in Tourism dx.doi.org/10.1080/13683500.2016.1225697

Pöyhönen, P. (1963a). A tentative model for the volume of trade between countries. WeltwirschaftlichesArchiv, 90, 93-99. 
Pöyhönen, P. (1963b). Toward a general theory of international trade. Ekonomiska Samfundets Tidskrift, 16, 69-78.

Reyna, S. (1994). Actas del seminario sobre el turismo rural en el desarrollo local, Laredo 2226 julio. Ministerio de Agricultura, Pesca y Alimentación, Madrid.

Ritchie, J. y Crouch, G. (2000). The competitiveness destinations: a susceptible perspective. Tourism Management, 21, 1-7.

Santeramo, F. y Morelli, M. (2015). Modelling tourism flows through gravity models: a quantile regression approach. Current Issues in Tourism. 19, 11, 1-7.

Song, H. y Li, G. (2008). Tourism Demand Modeling and Forecasting - A Review of Resent Research. Tourism Management, 29, 203-220.

Tinbergen, J. (1962). Shaping the world Economy: suggestions for international economic policy. New York: The Twentieth Century, Inc.

Vogt, M.G. (2008). Determinants of the Demand for US Exports and Imports of Tourism. Applied Economics, 40, 667-672.

Para citar este artículo: Martí Selva, L. y Puertas Medina, M. (2017). Caracterización del turismo español según el tipo de alojamiento: aproximación mediante un modelo de gravedad. Investigaciones Turísticas, (13), 77-95. http://dx.doi.org/10.461/INTURI2017.13.04 


\section{ANEXO}

Tabla A1. Resultados de los distintos métodos de estimación por zonas

\begin{tabular}{|c|c|c|c|c|c|c|c|c|c|c|c|c|}
\hline & \multicolumn{3}{|c|}{ Litoral Mediterráneo } & \multicolumn{3}{|c|}{ Costa Verde } & \multicolumn{3}{|c|}{ Archipiélago } & \multicolumn{3}{|c|}{ Interior Peninsular } \\
\hline & $\begin{array}{l}\text { Pooled } \\
\text { MCO }\end{array}$ & $\begin{array}{l}\text { Efectos } \\
\text { aleatorios }\end{array}$ & $\begin{array}{l}\text { Efectos } \\
\text { fijos }\end{array}$ & $\begin{array}{l}\text { Pooled } \\
\text { MCO }\end{array}$ & $\begin{array}{l}\text { Efectos } \\
\text { aleatorios }\end{array}$ & $\begin{array}{l}\text { Efectos } \\
\text { fijos }\end{array}$ & $\begin{array}{l}\text { Pooled } \\
\text { MCO }\end{array}$ & $\begin{array}{l}\text { Efectos } \\
\text { aleatorios }\end{array}$ & $\begin{array}{l}\text { Efectos } \\
\text { fijos }\end{array}$ & $\begin{array}{l}\text { Pooled } \\
\text { MCO }\end{array}$ & $\begin{array}{l}\text { Efectos } \\
\text { aleatorios }\end{array}$ & Efectos fijos \\
\hline $\mathrm{P}_{\mathrm{O}}$ & $1,094 * * *$ & $1,092 * * *$ & $-2,540$ & $0,978 * * *$ & $0,972 * * *$ & $5,275^{*}$ & $1,218 * * *$ & $1,205 * * *$ & $-7,898 * * *$ & $0,941 * * *$ & $0,939 * * *$ & $-11,588 * * *$ \\
\hline $\mathrm{P}_{\mathrm{D}}$ & $1,345 * * *$ & $1,355 * * *$ & $-10,583$ & $-0,028$ & $-0,144$ & $-0,528$ & $-1,615 * * *$ & $-1,876 * * *$ & $-5,939 * * *$ & $0,709 * * *$ & $0,711 * * *$ & $12,878^{* * *}$ \\
\hline $\mathrm{D}$ & $-0,803 * * *$ & $-0,824 * * *$ & --- & $-0,711 * * *$ & $-0,679 * * *$ & --- & $-0,238 * *$ & $-0,105$ & --- & $-0,544 * * *$ & $-0,533 * * *$ & --- \\
\hline $\mathrm{PIB}_{\mathrm{O}}$ & $1,050 * * *$ & $0,933 * * *$ & 0,740 & $2,085 * * *$ & $2,171 * * *$ & $-1,241$ & $1,968 * * *$ & $1,693 * * *$ & $-4,259 * * *$ & $1,676 * * *$ & $1,728 * * *$ & $-4,095$ \\
\hline $\mathrm{PIB}_{\mathrm{D}}$ & $-0,552 * * *$ & $-0,767 * * *$ & $-2,346$ & $-2,068 * * *$ & $-1,590 * * *$ & $4,805 * *$ & $-4,223 * * *$ & $-3,940 * * *$ & 1,638 & $-0,426 * *$ & $-0,390$ & $6,983 * *$ \\
\hline ITCT & $-0,265$ & $-0,537$ & 7,311 & $-1,999 * * *$ & $-1,932 * * *$ & $-3,945^{* *}$ & $-0,950$ & $-0,637$ & $8,719 * * *$ & $-0,709$ & $-0,463$ & $-0,888$ \\
\hline $\mathrm{C}$ & $0,521 * * *$ & $0,518 * * *$ & --- & $0,304 * * *$ & $0,313 * * *$ & --- & $1,183 * * *$ & $1,285 * * *$ & --- & $0,629 * * *$ & $0,632 * * *$ & --- \\
\hline $\mathrm{F}$ & $0,204 * * *$ & $0,189 * *$ & --- & $0.129 * * *$ & $0,141 * *$ & --- & --- & --- & --- & $0,347 * * *$ & $0,351 * * *$ & --- \\
\hline Chibar2 & \multicolumn{2}{|c|}{$427,93 * * *$} & & \multicolumn{2}{|c|}{$8,76^{* * *}$} & & \multicolumn{2}{|c|}{$299.74 * * *$} & & \multicolumn{2}{|c|}{$83,14 * * *$} & \\
\hline Chi2 & & \multicolumn{2}{|c|}{2,402} & & \multicolumn{2}{|c|}{2,669} & & \multicolumn{2}{|c|}{4,442} & & 1,560 & \\
\hline F-test & & & $45 * * *$ & & & $13,02 * * *$ & & & $65,57 * * *$ & & & $6,36 * * *$ \\
\hline
\end{tabular}

Notas:1) Chibar2 es el estadístico del test Breusch y Pagan que al salir significativo indica que es preferible el modelo de efectos aleatorios respecto al pooled.

2) Chi2 es el estadístico del test de Hausman que al salir no significativo indica que es preferible el modelo de efectos aleatorios respecto al de efectos fijos

3) F indica la prueba $F$ que al salir significativa es preferible usar el método de efectos fijos al modelo pooled.

4) ${ }^{* *}$ p-value $<0,05,{ }^{* * *} p$-value $<0,01$. 